\title{
Clinically oriented prediction of patient response to targeted and immunotherapies from the tumor transcriptome
}

\author{
Gal Dinstag ${ }^{1} \star$, Eldad D. Shulman ${ }^{1}$, Efrat Elis ${ }^{1}$, Doreen S. Ben-Zvi ${ }^{1}$, Omer Tirosh ${ }^{1}$, Eden Maimon ${ }^{1}$, Isaac \\ Meilijson ${ }^{1,2}$, Emmanuel Elalouf $^{1}$, Eyal Schiff ${ }^{1}$, Danh-Tai Hoang ${ }^{3}$, Sanju Sinha ${ }^{4}$, Nishanth Ulhas Nair ${ }^{4}$, \\ Joo Sang Lee ${ }^{5}$, Alejandro A. Schäffer ${ }^{4}$, Ze'ev Ronai ${ }^{6}$, Dejan Juric ${ }^{7,8}$, Andrea B. Apolo ${ }^{9}$, William L. \\ Dahut $^{9}$, Stanley Lipkowitz ${ }^{10}$, Raanan Berger ${ }^{11}$, Razelle Kurzrock ${ }^{12}$, Antonios Papanicolau-Sengos ${ }^{13}$, \\ Fatima Karzai ${ }^{9}$, Mark R. Gilbert ${ }^{14}$, Kenneth Aldape ${ }^{13}$, Padma S. Rajagopal ${ }^{4,10}$, Tuvik Beker ${ }^{1, \star}$, Eytan \\ Ruppin $^{4, *} \&$ Ranit Aharonov ${ }^{1, *}$ \\ ${ }^{1}$ Pangea Biomed Ltd., Tel Aviv 6971003, Israel \\ ${ }^{2}$ Tel Aviv University, Tel Aviv 69978, Israel \\ ${ }^{3}$ Biological Data Science Institute, College of Science, The Australian National University, Canberra, ACT, Australia. \\ ${ }^{4}$ Cancer Data Science Laboratory (CDSL), National Cancer Institute (NCI), National Institutes of Health (NIH), \\ Bethesda, MD, USA \\ ${ }^{5}$ Department of Precision Medicine, School of Medicine \& Department of Artificial Intelligence, Sungkyunkwan \\ University, Suwon, Republic of Korea \\ ${ }^{6}$ Cancer Center, Sanford Burnham Prebys Medical Discovery Institute, La Jolla, CA, USA. \\ ${ }^{7}$ Massachusetts General Hospital Cancer Center, Boston, MA, USA \\ ${ }^{8}$ Department of Medicine, Harvard Medical School, Boston, MA, USA \\ ${ }^{9}$ Genitourinary Malignancies Branch, Center for Cancer Research, National Cancer Institute, National Institutes of \\ Health, Bethesda, MD, USA \\ ${ }^{10}$ Women's Malignancies Branch, Center for Cancer Research, National Cancer Institute, Bethesda, MD, USA. \\ ${ }^{11}$ Cancer Center, Chaim Sheba Medical Center, Tel Hashomer 5262000, Israel \\ ${ }^{12}$ Worldwide Innovative Network (WIN) for Personalized Cancer Therapy \\ ${ }^{13}$ Laboratory of Pathology, Center for Cancer Research, National Cancer Institute, Bethesda, MD, USA. \\ ${ }^{14}$ Neuro-Oncology Branch, National Cancer Institute, National Institutes of Health, Bethesda, MD, USA. \\ *Equal corresponding authors: gal@pangeabiomed.com (G.D.), tuvik@pangeabiomed.com (T.B.), \\ evtan.ruppin@nih.gov (E.R.), ranit@pangeabiomed.com (R.A.)
}

\section{Abstract}

Precision oncology is gradually advancing into mainstream clinical practice, demonstrating significant survival benefits. However, eligibility and response rates remain limited in many cases, calling for better predictive biomarkers. Here we present ENLIGHT, a transcriptomics-based computational approach that identifies clinically relevant genomic interactions and uses them to predict patients' response to a variety of therapies in multiple cancer types without training on previous response data. We study ENLIGHT in two translationally oriented scenarios, Personalized Medicine (PM), aimed at prioritizing treatments for a single patient, and Clinical Trial Design (CTD), selecting the most likely responders in a 
patient cohort. Evaluating ENLIGHT's PM performance on 21 blinded clinical trial datasets, we show that it can effectively predict treatment response across multiple therapies and cancer types (obtaining an odds ratio of 2.59), substantially improving upon SELECT, a previously published transcriptomics-based approach, and performing as well as supervised predictors developed for specific indications and drugs, but on a much broader array of therapies and indications. In the CTD scenario, ENLIGHT can markedly enhance the success of clinical trials by excluding non-responders while achieving more than $90 \%$ of the response rate attainable under an optimal exclusion strategy. In sum, ENLIGHT is one of the first approaches to demonstrably predict therapeutic response across multiple cancer types by leveraging the transcriptome.

\section{Introduction}

The current paradigm of precision oncology, rooted in the 1990s development of trastuzumab and imatinib, focuses on matching individual targets to molecularly derived therapies ${ }^{1,2}$. In the past 20 years, cancer therapeutics, driven by treatments developed for specific oncogenes and by the advent of immunotherapy, has overwhelmingly dominated regulatory drug approvals ${ }^{3}$. For patients with a qualifying biomarker and otherwise limited treatment options, this paradigm can demonstrate improvement in clinical outcomes ${ }^{4-7}$, as in the I-PREDICT study ${ }^{8}$, which uses DNA biomarkers to identify novel combination therapies for patients. However, large studies such as $\mathrm{NCl}-\mathrm{MATCH}$ demonstrate the limitations of the variant-centered biomarker approach, with less than $20 \%$ of patients ultimately assigned to single-therapy treatment arms ${ }^{9}$. Response rates in the setting of targeted therapy also show broad ranges, between $25-75 \%$ of patients ${ }^{10}$. Although qualification for immunotherapy varies by cancer type, often only $20-40 \%$ of patients respond to treatment ${ }^{11,12}$.

One strategy to broaden which patients qualify for treatment and to improve response rates is to leverage omics data beyond genomics, with the main focus of current research being on transcriptomics. Clinical programs such as University of Michigan's MIONCOSEQ have sought to integrate DNA and RNA-based sequencing to validate and support existing biomarkers ${ }^{13,14}$. WINTHER was a prospective umbrella trial in which patients received personalized 
monotherapy or combination therapy based on genomic or transcriptomic data ${ }^{15}$. This was the first clinical trial to examine the utility of RNA sequencing (RNA-seq) in a prospective clinical setting by targeting highly expressed cancer-associated genes, demonstrating that the tumor transcriptome could be used to provide complementary clinical information to DNA.

Other attempts to identify predictors of response from the transcriptome have only been studied in limited contexts to date ${ }^{16-19}$. Notable examples include Sammut et al. ${ }^{20}$ who demonstrated the ability of RNA-seq to improve prediction of pathologic complete response in early breast cancer (a space where RNA-seq has already demonstrated clinical utility ${ }^{21-23}$ ) beyond DNA-seq, pathology imaging and clinical data; Jiang et al. ${ }^{17}$ and Cui et al. ${ }^{24}$ who built predictors for response to immune checkpoint blockade based on the tumor's immune microenvironment reflected by RNA data; and the OncoTarget / OncoTreat program at Columbia University that builds on using RNA-seq alone by mapping protein interaction networks derived from the transcriptome to prioritize cancer drivers for treatment and was evaluated in pancreatic neuroendocrine tumors ${ }^{25}$. These important and novel approaches have, however, been limited to highly specific clinical contexts and treatments and each has different data requirements. Using such approaches in a scenario where multiple treatment options per patient exist is therefore a very challenging task ${ }^{12,26-28}$.

In a recent effort to overcome these limitations and develop a uniform systematic approach for stratifying patients to multiple therapies based on the tumor transcriptome, Lee et $a l^{29}$ have demonstrated that synthetic lethality $(S L)$ and synthetic rescue (SR) interactions ${ }^{1}$ can be leveraged to predict treatment response via the transcriptome. Their framework, SELECT, mines large-scale in-vitro omics data and TCGA patient data to computationally infer putative pairs of Genetic Interactions (GIs) and uses this data to predict and prioritize a variety of treatments. It showed that tumor expression could be analyzed systematically to predict patients' response to a broad range of targeted therapies and immunotherapy in multiple

\footnotetext{
${ }^{1}$ An SL interaction between two genes means that the simultaneous inactivation of both genes reduces the viability of the cell while the individual inactivation of each does not ${ }^{30,31}$. An SR interaction is one in which the inactivation of one gene reduces cell viability, but the alteration of another gene's activity, termed the rescuer, restores (rescues) viability ${ }^{32,33}$.
} 
cancer types with high accuracy, beyond case-specific biomarkers. However, SELECT has a few fundamental limitations, which we have set to remedy.

To this end, here we substantially extend the work of Lee et al. and present ENLIGHT, a transcriptomics-based precision oncology pipeline based on the Gl-approach. First, we surveyed the ability of SELECT to build relevant GI networks for 105 FDA approved targeted and immunotherapies, finding that it could build relevant GI networks for only $67 \%(70 / 105)$ of them, thus limiting its use in clinical settings. Addressing this challenge with new Gl-based scores, ENLIGHT successfully extends the scope of SELECT to all these 105 therapies. Second, SELECT had focused on maximizing the area under the ROC curve (AUC) - a measure commonly used in data science but not directly applicable in clinical settings. In contrast, ENLIGHT's design and parameter optimization is aimed at maximizing its performance on three key translational tasks: (i) Drug coverage - determining the range of drugs for which predictions can be obtained and extending the prior range of SELECT; (ii) Personalized medicine - evaluating and prioritizing multiple candidate treatment options for individual patients. Since a variety of treatment options are considered in this scenario, a good test should primarily have a high Positive Predictive Value (PPV, also known as precision) taking priority over sensitivity (recall), which can be moderate; (iii) And finally, Improving Clinical Trial Design (CTD) by excluding patients that are unlikely to respond to the treatment by employing a test that has a high Negative Predictive Value (NPV).

To evaluate ENLIGHT's performance, we collected 21 new cohorts beyond those analyzed in the SELECT study, overall spanning 11 different drugs in 11 cancer indications, and kept them blinded for evaluation purposes. We find that while SELECT is reassuringly predictive on the subset of those datasets for which it could produce results, ENLIGHT still performs better when evaluated using clinically relevant performance measures. We show that (i) ENLIGHT produces predictions for all the drugs used in these cohorts; (ii) ENLIGHT scores are associated with better response (OR=2.59; 95\% confidence interval $[1.85,3.55]$; $p=3.41 e-8)$. (iii) ENLIGHT can be used to successfully exclude patients from clinical trials, quite strikingly achieving more than $90 \%$ of the response rate attainable under an optimal exclusion strategy for both immune 
therapies and targeted monoclonal antibody (mAb) treatments. Finally, we find that ENLIGHT performs as well as supervised tests developed for specific indications and drugs and better than other known transcriptomics-based predictive signatures. These results showcase the effectiveness of ENLIGHT as a tool to improve translational oncology research.

\section{Results}

\section{Tuning and Evaluation Datasets}

To tune the parameters for ENLIGHT, including the GI network size and a cutoff on the ENLIGHT Matching Score (EMS) that is used as a decision threshold for predicting favorable response (see below), we selected eight tuning sets - six datasets ${ }^{34-39}$ already analyzed in Lee et al. ${ }^{29}$ along with two other datasets ${ }^{40,41}$ from the public domain. These were selected as they span a range of different treatments, therapeutic classes, response rates and sample sizes, reflecting diverse real-world data, covering five targeted therapies and one immune checkpoint blockade (ICB) therapies.

To study the value of ENLIGHT on real world data, which was not previously analyzed by either SELECT or ENLIGHT, we surveyed the public domain (GEO, ArrayExpress, and the broader literature) for available datasets of patients receiving targeted or immunotherapies, containing both pre-treatment transcriptomics and response information. We additionally used an unpublished dataset that was obtained as part of a collaboration with Massachusetts General Hospital (MGH). Overall, we identified and set aside 21 datasets ${ }^{20,24,42-60}$ - the evaluation sets as unseen data for evaluation. See Table S1 and Supplementary Note 1 for full details.

\section{Overview of the ENLIGHT Pipeline}

ENLIGHT's drug response prediction pipeline generally follows the flow of SELECT ${ }^{29}$ which comprises two steps (Figure 1a): (i) Given a drug, the inference engine identifies the clinically 
relevant GI network of the drug's target genes. As was done in SELECT, we start with a list of initial candidate SL/SR pairs by analyzing cancer cell line dependencies with RNAi, CRISPR/Cas9, or pharmacological inhibition, based on the principle that SL/SR interactions should decrease/increase tumor cell viability, respectively, when 'activated' (e.g., in the SL case, when both genes are down-regulated). Among these candidate pairs, we select those that are more likely to be clinically relevant by analyzing a database of tumor samples with associated transcriptomics and survival data, requiring a significant association between the joint inactivation of target and partner genes and better patient survival for SL interactions, and analogously for SR interactions. Finally, among the candidate pairs that remain after these steps, we select those pairs that are supported by a phylogenetic profiling analysis. When considering combination therapies, ENLIGHT computes a GI network based on the union of all the drug targets. (ii) The drug-specific GI network is then used to predict a given patient's response to the drug based on the gene expression profile of the patient's tumor. The ENLIGHT matching score (EMS), which evaluates the match between patient and treatment, is based on the overall activation state of the genes in the drug target's GI network, reflecting the notion that a tumor would be more susceptible to a drug that induces more active SL interactions and fewer active SR interactions. An important prerequisite for a drug to be analyzed by this approach is that the gene targets are well defined. Thus, in this work we focus on targeted therapies and ICBs.

In developing ENLIGHT, we have extended and improved this basic framework, by introducing the following adaptations (Supplementary Note 2): (i) ENLIGHT's GI networks include both SL and SR interactions that are concomitantly inferred for each drug, thereby considerably increasing drug coverage relative to SELECT, which utilizes only one type of interaction per network (for immunotherapy, where SELECT had no coverage limitation, we use only SR interactions as was done in SELECT). (ii) To improve the inference engine, we follow the idea presented in Lee et al. ${ }^{61}$ and Sahu et $a l .{ }^{62}$ and add an additional step based on clinical data, requiring that the joint inactivation of a candidate SL pair is under-represented in tumors (and analogously for SR partners). Furthermore, the tests to select candidate SL/SR pairs based on survival analysis in clinical datasets were improved to increase robustness and statistical power. 
(iii) ENLIGHT GI networks are considerably larger than those of SELECT to reduce score variation across drugs and indications. (iv) To improve the prediction engine, for immunotherapy and other mAbs, which are highly target-specific, the EMS incorporates the expression of the target as an additional score component.

We emphasize that the GI networks are inferred independently of any treatment data. Notably, while the parameters of ENLIGHT, namely the network size and thresholds for the inference tests, were optimized on the tuning datasets, we used the same fixed sets of parameters to predict all treatment outcomes across all 21 evaluation sets. These procedures markedly mitigate the risk of deriving overfitted predictors that would not predict response in new datasets.

\section{Setting the ENLIGHT Decision Threshold for Personalized Medicine}

To define an ENLIGHT-based test for the personalized medicine scenario, we define a uniform decision threshold on the ENLIGHT Matching Score (EMS), above which the probability of a patient to respond is high. The chosen decision threshold should maximize the proportion of true responders among those predicted to respond (the PPV), while the proportion of true responders identified by the test (termed its sensitivity, or recall) does not have to be very high since multiple treatment options are considered. Importantly, the decision threshold set on the tuning cohorts is fixed and is used as is in evaluating ENLIGHT's performance on all cohorts and treatments. The EMS distributions in the tuning cohorts show that the EMS are significantly higher among responders compared to non-responders (Figure 1b), testifying to its discriminatory power. Figure $\mathbf{1 c}$ shows that a decision threshold of $\mathbf{0 . 5 4}$, which will be used henceforth, maximizes the PPV while still maintaining a reasonable sensitivity (for the complete cohort specific PPV and sensitivity curves see Figures S1 and S2). ENLIGHT's performance is evaluated using the odds ratio (OR) for response, which denotes the odds to respond when the treatment is recommended (ENLIGHT-matched, i.e., EMS above the decision threshold), divided by the odds when it's not. As shown in Figure 1b, ENLIGHT obtains an aggregate OR of 2.98 on 
the tuning cohorts (aggregation based on individual patients; 95\% confidence interval [Cl], 1.8 to $5 ; p=4 \mathrm{e}-05)$.

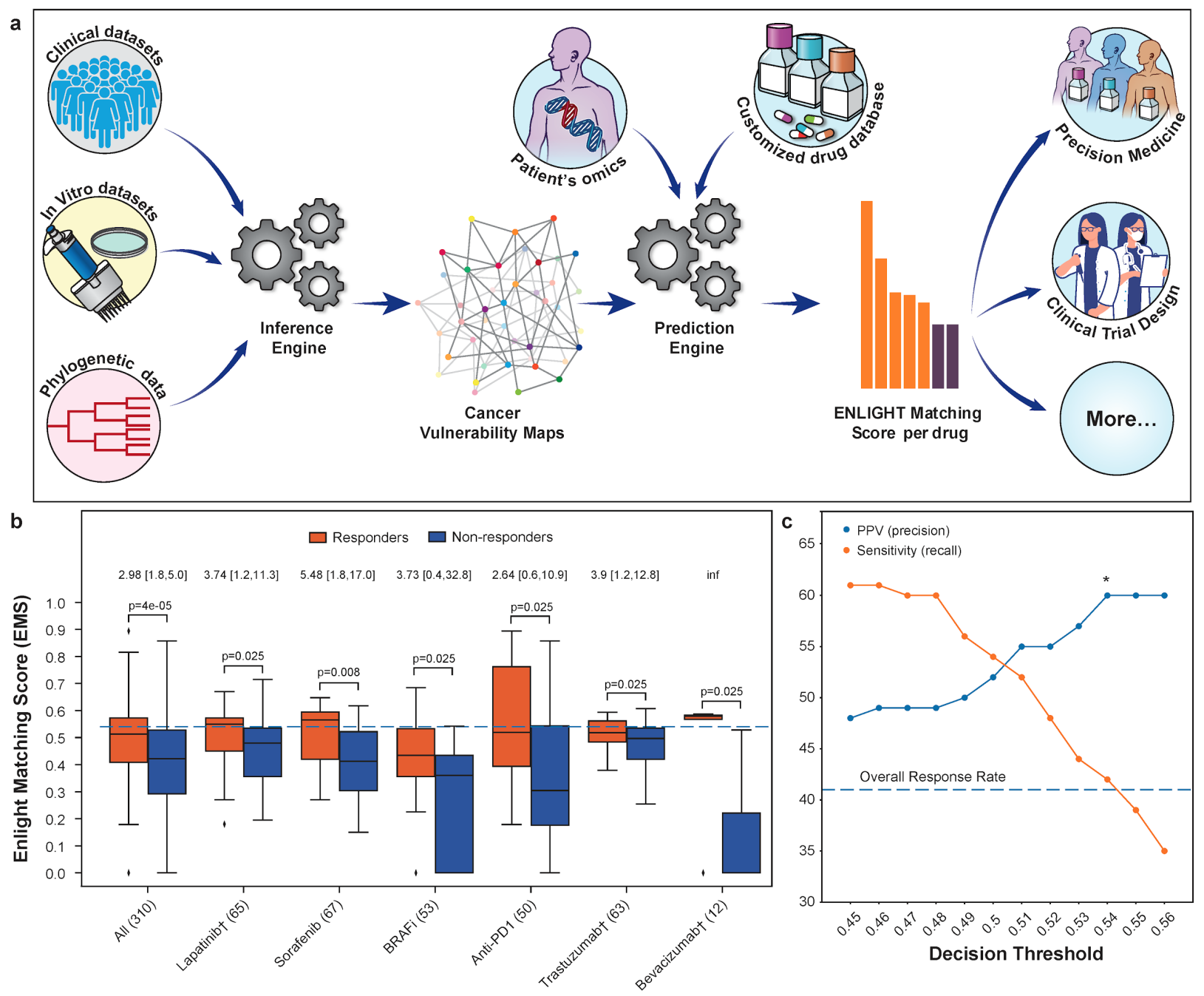

\section{Figure 1: The ENLIGHT Pipeline and its tuning for Personalized Medicine. (a)}

ENLIGHT's pipeline starts by inferring the genetic interaction network of a given drug. The GI network and omics data from patients are then used to predict treatment response. (b) ENLIGHT matching scores in responders (orange) are higher than in non-responders (blue), across the tuning cohorts. The $3 \mathrm{BRAFi}$ cohorts are very small, so their results are presented in aggregate. The 'All' column 
presents the results for all patients aggregated together. The $p$-value is based on the one-sided Mann-Whitney test. The horizontal line marks the EMS decision threshold. The odds ratio of ENLIGHT-matched cases appears on top of each pair of bars, with brackets indicating the $95 \%$ confidence interval. The number of patients in each cohort is provided in parentheses. (c) The PPV (precision) and sensitivity (recall) on the aggregate tuning cohort (y axis) as a function of the decision threshold ( $x$ axis).

\section{ENLIGHT Successfully Predicts Patients Treatment Response}

We next turned to evaluate how ENLIGHT performs in identifying the true responders in the 21 unseen patient cohorts that we have collected, spanning ICBs, mAbs, and targeted small molecules. Notably, the response data for all evaluation cohorts was unblinded only after finalizing the ENLIGHT pipeline including fixing the decision threshold and calculating EMS for all patients. Figure $2 \mathrm{a}$ shows that ENLIGHT-matched treatments are associated with better patient response $(O R>1)$ in all cohorts except for two (Sorafenib 2 and one ICB cohort) with an aggregate OR of 2.59 (95\% confidence interval [Cl], 1.89 to $3.55 ; p=3.41 e-8, \mathrm{~N}=697$ ). Correspondingly, Figure $\mathbf{2 b}$ shows that the overall PPV obtained for ENLIGHT-matched cases is markedly higher than the overall response rate (52\% vs $38 \%$, a $36.84 \%$ increase, $p=3.30$ e- 13 , one sided proportion test, and see Table $\mathbf{S 2}$ for a more detailed account). Interestingly, ENLIGHT is more accurate in immunotherapies and other mAbs vs. targeted small molecules, which aligns with its reliance on accurate drug targets. Notably, when a patient received a combination of targeted and chemotherapy agents (see cohorts marked with a cross), the EMS was calculated for the targeted agent alone, however, remarkably, the performance is still maintained. Supplementary Note 3 provides a detailed comparison of ENLIGHT's performance to that of its predecessor, SELECT, and to four widely used transcriptomic based biomarkers, on the 21 evaluation cohorts. Briefly, SELECT could identify Gls enabling prediction on 10 of these cohorts, and on those SELECT obtains an OR of $1.23([0.77,1.97], p=0.065)$ with a recall of $49 \%$, 
while ENLIGHT obtains an OR of 2.3 ([1.42,3.73], $p=0.0002)$ with a recall of 54\%. Moreover, ENLIGHT's performance is better than other known transcriptomics-based predictive signatures. Overall, these results provide strong support for the ability of ENLIGHT to provide clinical benefit in the precision medicine scenario, spanning a variety of different treatments and cancer types.

Except for tuning very few parameters on a tuning cohort, as described above, ENLIGHT is essentially an unsupervised prediction method that relies on a series of biologically motivated statistical tests that underlie the inference of Gls and their utilization, and does not train on treatment outcome data. We next turned to compare its predictive power to that of transcriptomics-based biomarker signatures generated via supervised machine learning on specific training cohorts. To this end, we have studied four of the evaluation datasets, for which a supervised classifier was presented in the original publication and data enabling us to perform this comparison was provided (Figure 2c). In one of these datasets, Sammut et al. ${ }^{20}$ used supervised learning to predict response to chemotherapy with or without trastuzumab among HER2+ breast cancer patients. We focused on the subset of 56 patients who received trastuzumab and for which the model's scores were published. ENLIGHT achieved a slightly higher AUC than that reported in the original study, without ever training on these data, nor on any other data of response to trastuzumab, and without using any clinical features. Similarly, Cui et $a .^{24}$ developed a biomarker for response to anti-PD1 in melanoma (see anti-PD1 ${ }_{5}$ in Table S1) and Raponi et al. ${ }^{52}$ and Raponi et al. ${ }^{53}$ developed biomarkers for response to tipifarnib in acute myeloid leukemia (AML) to which we compared ENLIGHT's performance. Here again, ENLIGHT's predictive performance is comparable to that of biomarkers developed using a supervised approach for a specific treatment and indication. Taken together, these results demonstrate the remarkable predictive power of ENLIGHT as a pan-cancer and pan-treatment response predictor, which successfully competes with supervised models specifically trained for narrow predictive scenarios. 
Finally, we evaluated ENLIGHT as a personalized medicine tool in a multi-arm clinical trial setting, by analyzing data from the WINTHER trial, a large-scale prospective clinical trial that has incorporated genetic and transcriptomic data for cancer therapy decision making in adult patients with advanced solid tumors ${ }^{15}$. ENLIGHT was able to provide predictions for all patients, except four (see Supplementary Note 4). The EMS of the responders were significantly higher than those of non-responders ( $p=4 \mathrm{e}-04$, Figure $2 \mathrm{~d}$ ). The odds ratio of ENLIGHT-matched treatments is 11.15 ( $p=8 \mathrm{e}-04$, Figure $2 \mathrm{~d}$ ), and the PPV is more than two times higher than the overall response rate (Figure 2e). Further analysis shows that responders had significantly higher EMS than non-responders also for the 24 patients treated with a combination of drugs (Figure S4) and that ENLIGHT-matched treatments were associated with better response, without being hampered by the background of chemotherapy treatment (Table S3). In comparison, SELECT's response score was predictive of response but failed to provide coverage (i.e., treatment recommendations) to about $30 \%$ of the patients, and did not offer a decision threshold for determining response. 
bioRxiv preprint doi: https://doi.org/10.1101/2022.02.27.481627; this version posted March 1, 2022. The copyright holder for this preprint (which was not certified by peer review) is the author/funder. All rights reserved. No reuse allowed without permission.

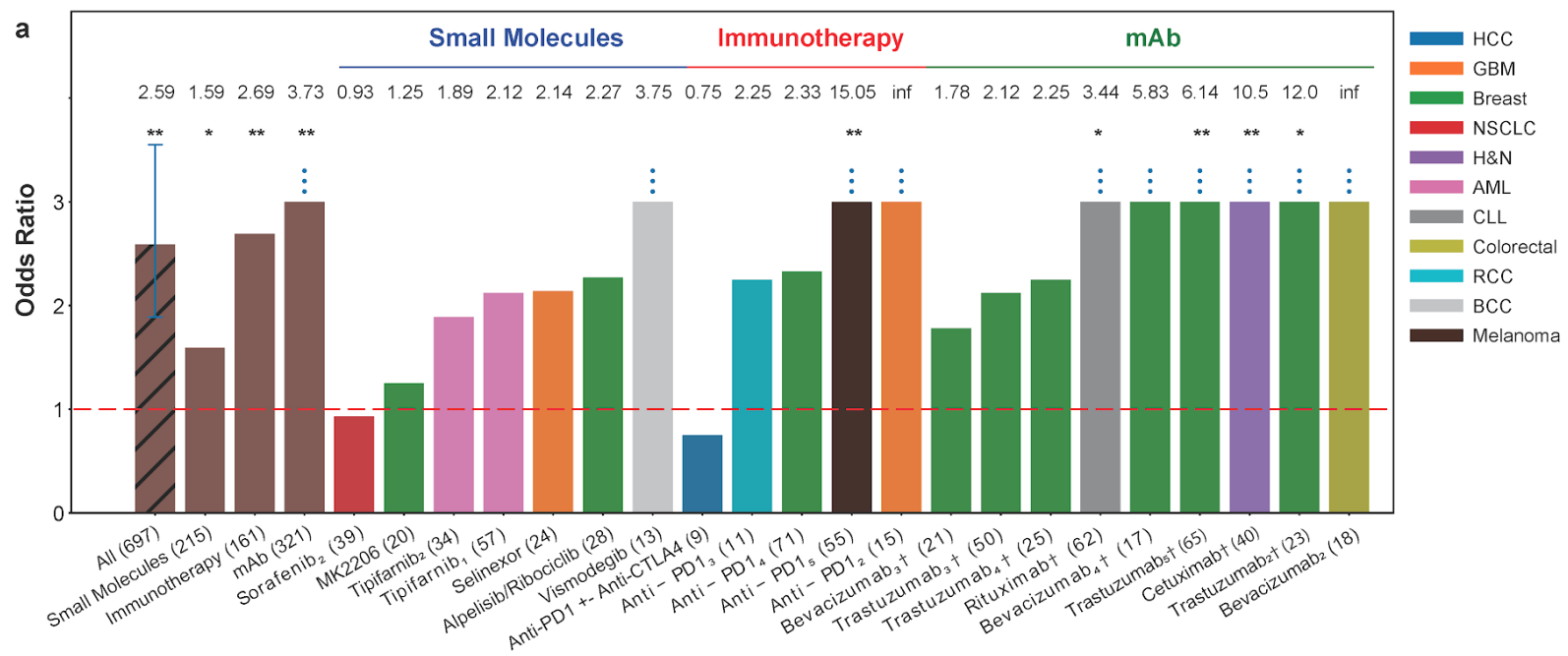

b
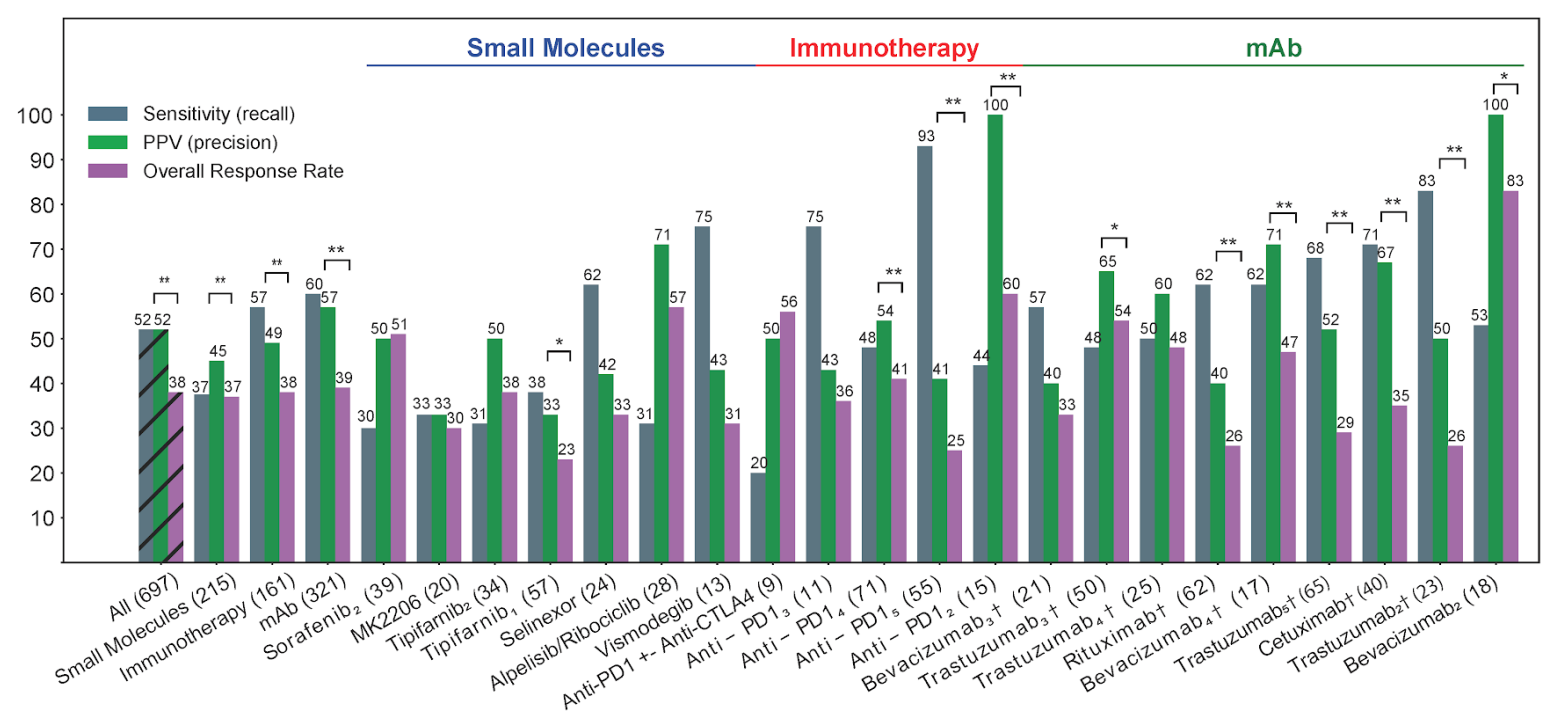

c

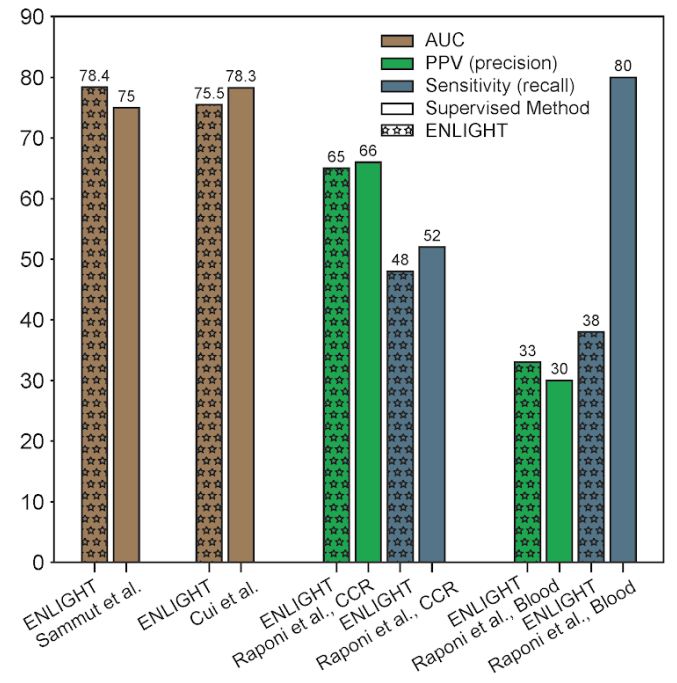

d

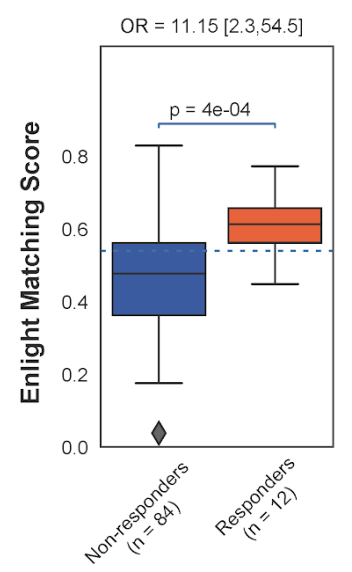

e

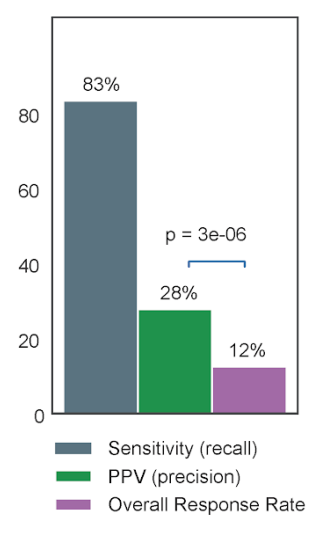


Figure 2: ENLIGHT's ability to stratify patients for therapy (a) The bar graphs show the odds ratio for response of ENLIGHT-matched cases in the 21 evaluation cohorts (OR values appear on top of each bar; all eight patients predicted to respond in the Bevacizumab ${ }_{2}$ cohort responded to the treatment, resulting in an infinite OR). (b) ENLIGHT's sensitivity and PPV vs. the overall response rate for the evaluation cohorts. (c) Comparison of ENLIGHT's performance to that of biomarkers developed using supervised methods. (d) In the WINTHER trial, responders (orange) have significantly higher EMS than non-responders (blue); $\mathrm{p}$-value is based on one-sided Mann-Whitney test. The horizontal line marks the decision threshold. (e) ENLIGHT's sensitivity and PPV vs. overall response rate in the WINTHER trial. [ $\dagger$ : Patients in these cohorts received a combination of targeted and chemotherapy; $\left.{ }^{*}: \mathrm{p}<0.1 ; * *: p<0.05\right]$

\section{ENLIGHT Enables near optimal exclusion of non-responding patients in Clinical Trial Design}

In the Clinical Trial Design scenario we are interested in identifying a sub-population of non-responding patients who could be excluded from the trial a priori, thereby allowing smaller studies to achieve higher response rates with adequate statistical power. Figure $\mathbf{3}$ (top row) depicts the NPV of ENLIGHT as a function of the percent of patients excluded, where patients are excluded by order of increasing EMS. For both ICB and other mAbs, ENLIGHT's NPV curve is considerably higher than the NPV expected by chance, i.e., the percentage of non-responders, testifying to its benefit. For targeted small molecules, however, it is unable to reliably identify non-responders, an issue that should be further studied and improved upon in future work.

The bottom row of Figure 3 depicts the response rate in the remaining cohort after excluding patients with EMS below the decision threshold. As evident, ENLIGHT-based exclusion considerably increases the response rate among the remaining patients (middle, solid line). The 
dotted-dashed line represents the limit performance of an optimal "all-knowing" classifier that excludes all non-responders, retaining only true responders (correspondingly, the $x$ axes end when this optimal classifier excludes all true non-responders, achieving the optimal response rate of $100 \%$ ). Focusing on a practical exclusion range of up to $25 \%$ of the patients (shaded area), ENLIGHT-based exclusion achieves $89 \%-96 \%$ and $90 \%-100 \%$ of the optimal exclusion response rate, for both ICB and other mAbs, respectively. It is important to acknowledge that the ENLIGHT-based exclusion strategy assumes knowledge of the EMS distribution in the trial, which may not be known a priori, but could be estimated using historical transcriptomics data from a reference population of the pertaining cancer indication and clinical characteristics.
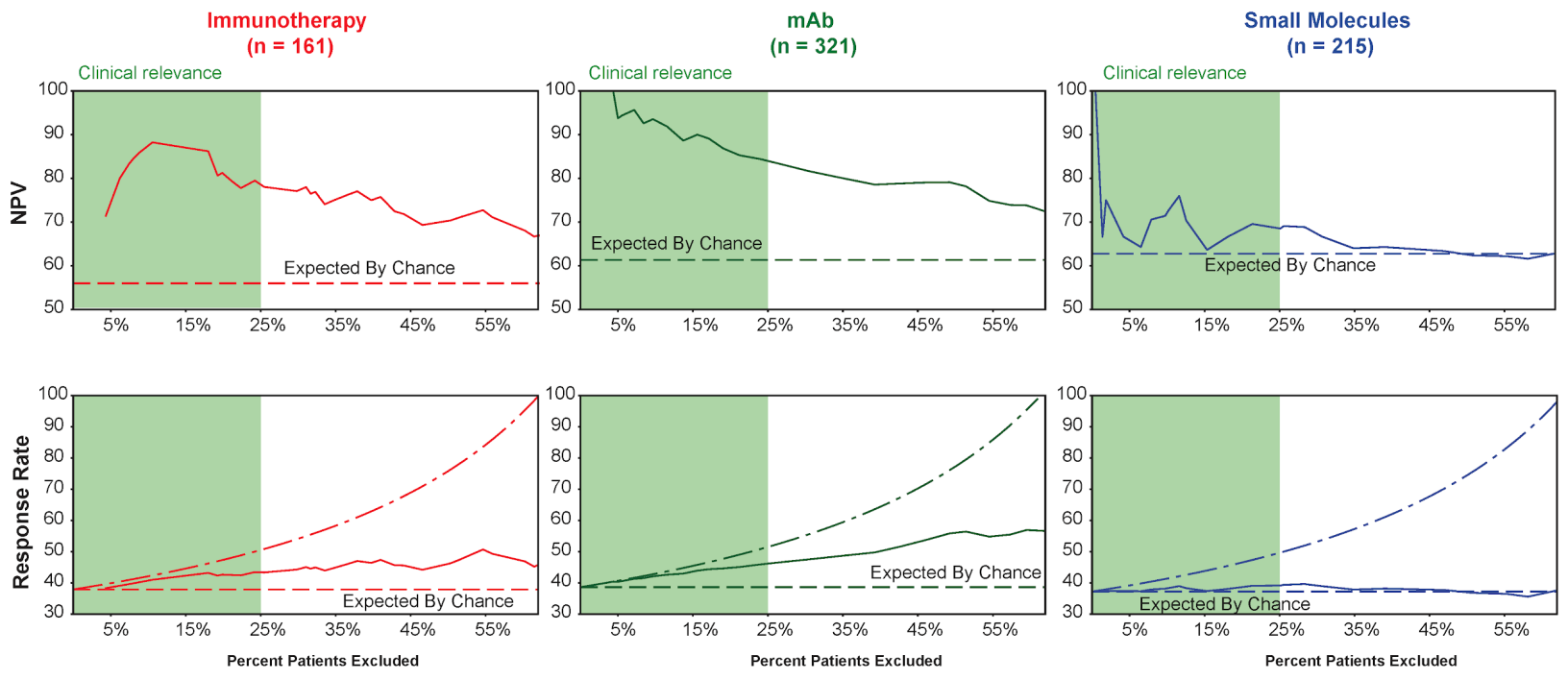

Figure 3: ENLIGHT can facilitate the exclusion of non-responding patients in

Clinical Trials. Each of the three columns depicts ENLIGHT's results on the aggregate of all evaluation cohorts from a given therapeutic class. Panels on the top row display the NPV (percent of true non-responders out of those predicted as non-responders) as a function of the percentage of patients excluded. The horizontal line denotes the actual percent of non-responders in the corresponding aggregate cohort (i.e., the NPV expected by chance). Panels on the bottom row display the response rate among the remaining patients ( $y$ axis) after excluding a certain percentage of the patients (x axis). The horizontal line denotes the overall 
response rate in the aggregate cohort. The dotted-dashed line represents the upper bound on the response rate, achieved by the "all knowing" optimal classifier excluding only true non-responders.

\section{Discussion}

Here we present ENLIGHT, an algorithmic platform that leverages synthetic lethality and rescue interactions to predict response to targeted therapies and immunotherapies. ENLIGHT is an unsupervised approach that, like its predecessor, SELECT, leverages large-scale data in cancer to infer GI networks associated with drug targets on a whole genome scale, and then uses the activation patterns of the genes comprising these networks, as measured in the tumor, to generate a matching score for each possible treatment.

In contrast to its predecessor, ENLIGHT has been designed and evaluated with two real-world clinical scenarios in mind: personalized medicine, where one matches the best treatment to a patient based on a fixed decision threshold, and clinical trial design, where the goal is to a priori exclude non-responding patients in the best possible manner. Testing ENLIGHT on 21 unseen clinical cohorts showed that patients whose treatments were recommended by ENLIGHT have markedly better odds of response than the others. ENLIGHT is a first of its kind systematic approach that has clinically relevant benefits across a broad array of treatments and indications. Despite this broad applicability, its performance is comparable to that of supervised classifiers trained and predictive on very narrow and specific treatment cohorts. This comparison is of much interest, since, in theory, given sufficiently large datasets, such supervised methods are expected to yield higher performance on unseen datasets than unsupervised methods like ENLIGHT. However, if the training sets available are small (as is regrettably the case with many of the datasets currently available), practice may differ from theory and supervised methods may overfit and underperform. Finally, ENLIGHT can enhance 
clinical trial design, by efficiently excluding non-responsive patients while attaining $90 \%$ of the maximum attainable response rate in targeted mAbs and ICB cohorts.

In the past five years, bulk RNA-seq has become increasingly available and reliable, opening the door for translational and clinical applications ${ }^{63}$. The Pan-Cancer Analysis of Whole Genomes demonstrated how the majority of classical driver genes exhibit alterations that were potentially better characterized via RNA than DNA ${ }^{64}$. Beyond developing drugs for new targets, we must consider ways to expand eligibility criteria for existing targeted therapies and immunotherapies. Data from ENLIGHT can provide a biologically informed basis to test strongly prioritized off-label therapies for specific patient populations through Phase II trials. This may be especially helpful in rare cancer types or challenging clinical scenarios, where hypothesis generation will only require initial RNA-seq samples. Second, building on the "Clinical Trial Design" capacity of ENLIGHT, the tool can offer simulations focused on a given therapeutic option and its performance across multiple types of patient populations-a computational version of the resource-challenging yet exciting basket trial design.

The current study mainly establishes ENLIGHT's power to predict monotherapies. However, in clinical practice, identifying combination therapy is often desirable. Notably in this context, ENLIGHT shows promising results when predicting response to targeted therapy on the background of chemotherapy, suggesting that a clinician could use ENLIGHT to identify favorable immune/targeted therapy and combine it with chemotherapy as deemed fit clinically. ENLIGHT also shows promising initial results for predicting combinations of targeted therapies, but this was demonstrated only on a small sample and needs to be corroborated as more data accumulate.

Like any response prediction approach, ENLIGHT has several limitations that should be acknowledged. First, as it operates on the drug target level, it has very limited utility in predicting response to chemotherapies, and more generally, its prediction accuracy depends on the accuracy with which the targets of a given drug have been identified. While current applications of ENLIGHT have focused on bulk tumor transcriptomics, future work is needed to 
study its application to analyze single-cell tumor transcriptomics, to build predictors that consider the heterogeneity of tumors, and the important interplay between tumor and its immune microenvironment.

While ENLIGHT's performance is evaluated here on an unprecedented scope of unseen clinical trial datasets, its performance should be further evaluated in prospective studies. Indeed, based on the results obtained so far by SELECT and ENLIGHT, the design of such a multi-arm study - SYNTHESIS - is now underway at the National Cancer Institute's Center for Cancer Research. We hope that the results presented here will elicit additional prospective studies in other clinical centers across the globe.

\section{References}

1. Schwartzberg, L., Kim, E. S., Liu, D. \& Schrag, D. Precision Oncology: Who, How, What, When, and When Not? American Society of Clinical Oncology Educational Book (2018) doi:10.1200/EDBK_174176.

2. Deborah B. Doroshow, J. H. D. Genomics and the History of Precision Oncology. Surg. Oncol. Clin. N. Am. 29, 35 (2020).

3. Olivier, T., Haslam, A. \& Prasad, V. Anticancer Drugs Approved by the US Food and Drug Administration From 2009 to 2020 According to Their Mechanism of Action. JAMA Netw Open 4, e2138793-e2138793 (2021).

4. Quinn, R., Patel, R., Sison, C., Singh, A. \& Zhu, X. H. Impact of Precision Medicine on Clinical Outcomes: A Single-Institution Retrospective Study. Front. Oncol. 11, (2021). 
5. Cutler, D. M. Early Returns From the Era of Precision Medicine. JAMA 323, 109-110 (2020).

6. Paez, J. G. et al. EGFR mutations in lung cancer: correlation with clinical response to gefitinib therapy. Science 304, 1497-1500 (2004).

7. Davies, H. et al. Mutations of the BRAF gene in human cancer. Nature 417, 949-954 (2002).

8. Sicklick, J. K. et al. Molecular profiling of cancer patients enables personalized combination therapy: the I-PREDICT study. Nat. Med. 25, 744-750 (2019).

9. Flaherty, K. T. et al. Molecular Landscape and Actionable Alterations in a Genomically Guided Cancer Clinical Trial: National Cancer Institute Molecular Analysis for Therapy Choice (NCI-MATCH). J. Clin. Oncol. 38, 3883-3894 (2020).

10. Gyawali, B., D’Andrea, E., Franklin, J. M. \& Kesselheim, A. S. Response Rates and Durations of Response for Biomarker-Based Cancer Drugs in Nonrandomized Versus Randomized Trials. J. Natl. Compr. Canc. Netw. 18, 36-43 (2020).

11. Sharma, P., Hu-Lieskovan, S., Wargo, J. A. \& Ribas, A. Primary, Adaptive, and Acquired Resistance to Cancer Immunotherapy. Cell 168, 707-723 (2017).

12. Topalian, S. L., Taube, J. M., Anders, R. A. \& Pardoll, D. M. Mechanism-driven biomarkers to guide immune checkpoint blockade in cancer therapy. Nat. Rev. Cancer 16, 275-287 (2016).

13. Roychowdhury, S. et al. Personalized Oncology Through Integrative High-Throughput Sequencing: A Pilot Study. Sci. Transl. Med. 3, 111ra121 (2011).

14. Roychowdhury, S. \& Chinnaiyan, A. M. Translating cancer genomes and transcriptomes for precision oncology. CA Cancer J. Clin. 66, 75-88 (2016).

15. Rodon, J. et al. Genomic and transcriptomic profiling expands precision cancer medicine: 
the WINTHER trial. Nat. Med. 25, 751-758 (2019).

16. Gene expression profiling predicts clinical outcome of breast cancer. Nature (2002) doi:10.1038/news020128-6.

17. Jiang, P. et al. Signatures of T cell dysfunction and exclusion predict cancer immunotherapy response. Nat. Med. 24, 1550-1558 (2018).

18. Ayers, M. et al. IFN-Y-related mRNA profile predicts clinical response to PD-1 blockade. J. Clin. Invest. 127, 2930-2940 (2017).

19. Slamon, D. J. et al. Use of chemotherapy plus a monoclonal antibody against HER2 for metastatic breast cancer that overexpresses HER2. N. Engl. J. Med. 344, 783-792 (2001).

20. Sammut, S.-J. et al. Multi-omic machine learning predictor of breast cancer therapy response. Nature 601, 623-629 (2022).

21. Sparano, J. A. et al. Adjuvant Chemotherapy Guided by a 21-Gene Expression Assay in Breast Cancer. N. Engl. J. Med. 379, 111-121 (2018).

22. Cardoso, F. et al. 70-Gene Signature as an Aid to Treatment Decisions in Early-Stage Breast Cancer. N. Engl. J. Med. 375, 717-729 (2016).

23. National Comprehensive Cancer Network. Breast Cancer. https://www.nccn.org/professionals/physician_gls/pdf/breast.pdf (Accessed Feb, 15 2021).

24. Cui, C. et al. Ratio of the interferon-y signature to the immunosuppression signature predicts anti-PD-1 therapy response in melanoma. NPJ Genom Med 6, 7 (2021).

25. Alvarez, M. J. et al. A precision oncology approach to the pharmacological targeting of mechanistic dependencies in neuroendocrine tumors. Nat. Genet. 50, 979 (2018).

26. Tsimberidou, A. M., Fountzilas, E., Nikanjam, M. \& Kurzrock, R. Review of precision cancer 
medicine: Evolution of the treatment paradigm. Cancer Treat. Rev. 86, 102019 (2020).

27. Malone, E. R., Oliva, M., Sabatini, P. J. B., Stockley, T. L. \& Siu, L. L. Molecular profiling for precision cancer therapies. Genome Med. 12, 1-19 (2020).

28. Feng, F., Shen, B., Mou, X., Li, Y. \& Li, H. Large-scale pharmacogenomic studies and drug response prediction for personalized cancer medicine. J. Genet. Genomics 48, 540-551 (2021).

29. Lee, J. S. et al. Synthetic lethality-mediated precision oncology via the tumor transcriptome. Cell 184, 2487-2502.e13 (2021).

30. Shen, J. P. \& Ideker, T. Synthetic Lethal Networks for Precision Oncology: Promises and Pitfalls. J. Mol. Biol. 430, 2900-2912 (2018).

31. Lord, C. J. \& Ashworth, A. PARP inhibitors: Synthetic lethality in the clinic. Science 355, 1152-1158 (2017).

32. Velimezi, G. et al. Map of synthetic rescue interactions for the Fanconi anemia DNA repair pathway identifies USP48. Nat. Commun. 9, 2280 (2018).

33. Eischen, C. M., Woo, D., Roussel, M. F. \& Cleveland, J. L. Apoptosis triggered by Myc-induced suppression of $\mathrm{Bcl}-\mathrm{X}(\mathrm{L})$ or $\mathrm{Bcl}-2$ is bypassed during lymphomagenesis. Mol. Cell. Biol. 21, 5063-5070 (2001).

34. Pinyol, R. et al. Molecular predictors of prevention of recurrence in HCC with sorafenib as adjuvant treatment and prognostic factors in the phase 3 STORM trial. Gut 68, 1065-1075 (2019).

35. Dieci, M. V. et al. Integrated evaluation of PAM50 subtypes and immune modulation of pCR in HER2-positive breast cancer patients treated with chemotherapy and HER2-targeted 
agents in the CherLOB trial. Ann. Oncol. 27, 1867-1873 (2016).

36. Guarneri, V. et al. Prospective Biomarker Analysis of the Randomized CHER-LOB Study Evaluating the Dual Anti-HER2 Treatment With Trastuzumab and Lapatinib Plus Chemotherapy as Neoadjuvant Therapy for HER2-Positive Breast Cancer. Oncologist 20, 1001-1010 (2015).

37. Kakavand, H. et al. PD-L1 Expression and Immune Escape in Melanoma Resistance to MAPK Inhibitors. Clin. Cancer Res. 23, 6054-6061 (2017).

38. Rizos, H. et al. BRAF inhibitor resistance mechanisms in metastatic melanoma: spectrum and clinical impact. Clin. Cancer Res. 20, 1965-1977 (2014).

39. Riaz, N. et al. Tumor and Microenvironment Evolution during Immunotherapy with Nivolumab. Cell 171, 934-949.e16 (2017).

40. Watanabe, T. et al. Gene expression signature and response to the use of leucovorin, fluorouracil and oxaliplatin in colorectal cancer patients. Clin. Transl. Oncol. 13, 419-425 (2011).

41. Prat, A. et al. Research-based PAM50 subtype predictor identifies higher responses and improved survival outcomes in HER2-positive breast cancer in the NOAH study. Clin. Cancer Res. 20, 511-521 (2014).

42. Pentheroudakis, G. et al. A study of gene expression markers for predictive significance for bevacizumab benefit in patients with metastatic colon cancer: a translational research study of the Hellenic Cooperative Oncology Group (HeCOG). BMC Cancer 14, 111 (2014).

43. Birkbak, N. J. et al. Overexpression of BLM promotes DNA damage and increased sensitivity to platinum salts in triple-negative breast and serous ovarian cancers. Ann. Oncol. 29, 
903-909 (2018).

44. Verstraete, M. et al. Combining bevacizumab and chemoradiation in rectal cancer.

Translational results of the AXEBeam trial. Br. J. Cancer 112, 1314-1325 (2015).

45. Byers, L. A. et al. An epithelial-mesenchymal transition gene signature predicts resistance to EGFR and PI3K inhibitors and identifies Axl as a therapeutic target for overcoming EGFR inhibitor resistance. Clin. Cancer Res. 19, 279-290 (2013).

46. Dieci, M. V. et al. Integrated evaluation of PAM50 subtypes and immune modulation of pCR in HER2-positive breast cancer patients treated with chemotherapy and HER2-targeted agents in the CherLOB trial. Ann. Oncol. 27, 1867-1873 (2016).

47. Liu, J. C. et al. Seventeen-gene signature from enriched Her2/Neu mammary tumor-initiating cells predicts clinical outcome for human HER2+:ERa- breast cancer. Proc. Natl. Acad. Sci. U. S. A. 109, 5832-5837 (2012).

48. Shen, K. et al. Cell line derived multi-gene predictor of pathologic response to neoadjuvant chemotherapy in breast cancer: a validation study on US Oncology 02-103 clinical trial. BMC Med. Genomics 5, 51 (2012).

49. Bossi, P. et al. Functional Genomics Uncover the Biology behind the Responsiveness of Head and Neck Squamous Cell Cancer Patients to Cetuximab. Clin. Cancer Res. 22, 3961-3970 (2016).

50. Lassman, A. B. et al. A Phase II Study of the Efficacy and Safety of Oral Selinexor in Recurrent Glioblastoma. Clin. Cancer Res. 28, 452-460 (2022).

51. Magbanua, M. J. M. et al. Circulating tumor DNA and magnetic resonance imaging to predict neoadjuvant chemotherapy response and recurrence risk. NPJ Breast Cancer 7, 32 
(2021).

52. Raponi, M. et al. Identification of molecular predictors of response in a study of tipifarnib treatment in relapsed and refractory acute myelogenous leukemia. Clin. Cancer Res. 13, 2254-2260 (2007).

53. Raponi, M. et al. A 2-gene classifier for predicting response to the farnesyltransferase inhibitor tipifarnib in acute myeloid leukemia. Blood 111, 2589-2596 (2008).

54. Foà, R. et al. Chlorambucil plus rituximab with or without maintenance rituximab as first-line treatment for elderly chronic lymphocytic leukemia patients. American Journal of Hematology vol. 89 480-486 (2014).

55. Zhao, J. et al. Immune and genomic correlates of response to anti-PD-1 immunotherapy in glioblastoma. Nat. Med. 25, 462-469 (2019).

56. Hsu, C.-L. et al. Exploring Markers of Exhausted CD8 T Cells to Predict Response to Immune Checkpoint Inhibitor Therapy for Hepatocellular Carcinoma. Liver Cancer 10, 346-359 (2021).

57. Ascierto, M. L. et al. The Intratumoral Balance between Metabolic and Immunologic Gene Expression Is Associated with Anti-PD-1 Response in Patients with Renal Cell Carcinoma. Cancer Immunol Res 4, 726-733 (2016).

58. Pusztai, L. et al. Durvalumab with olaparib and paclitaxel for high-risk HER2-negative stage II/III breast cancer: Results from the adaptively randomized I-SPY2 trial. Cancer Cell 39, 989-998.e5 (2021).

59. Atwood, S. X. et al. Smoothened variants explain the majority of drug resistance in basal cell carcinoma. Cancer Cell 27, 342-353 (2015). 
60. Pinyol, R. et al. Molecular predictors of prevention of recurrence in HCC with sorafenib as adjuvant treatment and prognostic factors in the phase 3 STORM trial. Gut 68, 1065-1075 (2019).

61. Lee, J. S. et al. Harnessing synthetic lethality to predict the response to cancer treatment. Nat. Commun. 9, 1-12 (2018).

62. Sahu, A. D. et al. Genome-wide prediction of synthetic rescue mediators of resistance to targeted and immunotherapy. Mol. Syst. Biol. 15, e8323 (2019).

63. Byron, S. A., Van Keuren-Jensen, K. R., Engelthaler, D. M., Carpten, J. D. \& Craig, D. W. Translating RNA sequencing into clinical diagnostics: opportunities and challenges. Nat. Rev. Genet. 17, 257-271 (2016).

64. Calabrese, C. et al. Genomic basis for RNA alterations in cancer. Nature 578, 129-136 (2020). 